\title{
Influence of main design parameters of ship propeller shaft water-lubricated bearings on their properties
}

\author{
Wojciech Litwin, Ph. D. \\ Gdansk University of Technology
}

\begin{abstract}

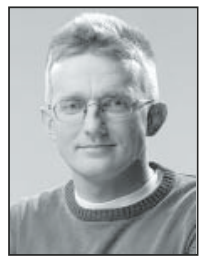

In recent years more and more frequently can be met ships in which propeller shaft water-lubricated polymer bearings have been applied. It results from their simplicity and associated relatively low initial and operational cost as compared with the complex classical sealed system based on oil-lubricated bearings. It is worth mentioning that the water-lubricated bearings are also environmentally friendly as no risk of pollution with lubricating oil used in classical systems, is involved. Design procedure of bearings in question based on materials made available by producers is relatively simple. However it turns out that choice of an appropriate sliding material, clearance value and optimum arrangement of lubricating grooves may greatly influence bearing's properties. As results from the performed research investigations, bearing of the kind is a highly sensitive unit. Problems of choosing a suitable bearing clearance, designing an optimum bush geometry, selecting a proper bush material are crucial for life time of the bearing. In particular the problem is in forming hydrodynamical properties of bearing as owing to its operation in the fluid friction regime durability of bearings, the very responsible units of propulsion transmission system, can be greatly extended.
\end{abstract}

Keywords: water lubrictaed bearings; stern tube bearings; sliding bearings

\section{INTRODUCTION}

Increasing ecological awareness, more and more stringent requirements for environmental protection, comprehensive control and strict attitude of institutions responsible for monitoring environmental state of waters, and finally cost calculations have made that increasing attention is paid to clean, environmentally safe design solutions.

In recent years many novel materials have been elaborated which are more and more willingly applied to building modern ocean engineering units. Also, novel water-lubricated sliding polymers have been created. Some of them have been approved by ship classification societies for shipbuilding application.

One of the main advantages of water-lubricated bearings of ship propeller shafts is their design simplicity and resulting relatively lower cost as compared with the classical system based on expensive oil-lubricated bearings and complex sealing units. The next obvious advantage of water-lubricated bearings is lack of risk of environmental pollution by lubricating medium.

However the water-lubricated bearings fitted with polymer bushes have also some limitations. The problem is to obtain stable hydrodynamic lubrication because of low viscosity of water. As results from the investigations have been performed so far it is possible though values of lubricating film thickness rarely exceed $10 \mu \mathrm{m}$. Hence such bearings are very sensitive to errors in bush shape and shaft position. The greatest hazard results from shaft misalignment also called as skewness of shaft axis [1].

\section{DESCRIPTION OF THE PROBLEM}

Sliding bearings lubricated with low-viscosity liquids, e.g. water, are usually very sensitive units. The reason is that if such bearing operates in the fluid friction regime its hydrodynamical load-carrying capacity will be much lower than that of a comparable classical oil-lubricated bearing. It results from low viscosity of the lubricating medium. As already mentioned, such bearings operate at minimum values of lubricating film thickness of the order of only a few micrometers, usually not acceptable for classical bearings even in view of height of unevenness in journal and bush. It should be remembered that at such low lubricating film thicknesses operational conditions of the bearing are influenced by many factors such as errors in shaft position and bush shape. Additionally, is important state of surface of sliding elements and operational conditions such as sliding velocity and pressure which decide on whether the bearing would operate under fluid friction or not. Results of the performed experimental tests have proved that the running-in process of the water-lubricated bearings fitted with polymer bush develops relatively fast. It means that as quickly as after 
a few hours of operation the bearing operational characteristics becomes distinctly changed (Fig. 1). Measurement results of shaft axis trajectory and pressure distribution in lubricating film confirm that in the typical marine sliding bearings fluid friction really occurs [1].

Designing a sliding bearing operating under fluid friction one tries to obtain a demanded value of hydrodynamical loadcarrying capacity at maintaining certain margin so as to keep - in a failure situation when the load increases and film thickness decreases - the bearing operating still under fluid friction. This is very important because of safety reasons as well as of necessity to increase life time of the bearing, significantly. The described effect of the maintaining of certain margin of load-carrying capacity may be difficult to achieve in slide bearings lubricated with low-viscosity liquids. It results from the much lower maximum load-carrying capacity of the value estimated to be within the range of $0.3 \div 0.5 \mathrm{MPa}$, depending on a kind of material.

Therefore in designing a sliding bearing lubricated with a low-viscosity liquid and intended for long and reliable operation one should design it for operation in the fluid friction regime and with a possibly large margin of load-carrying capacity. The design parameters of the bearing such as its size, clearance value, kind of bush material should be taken into account and lubricating grooves arranged appropriately.

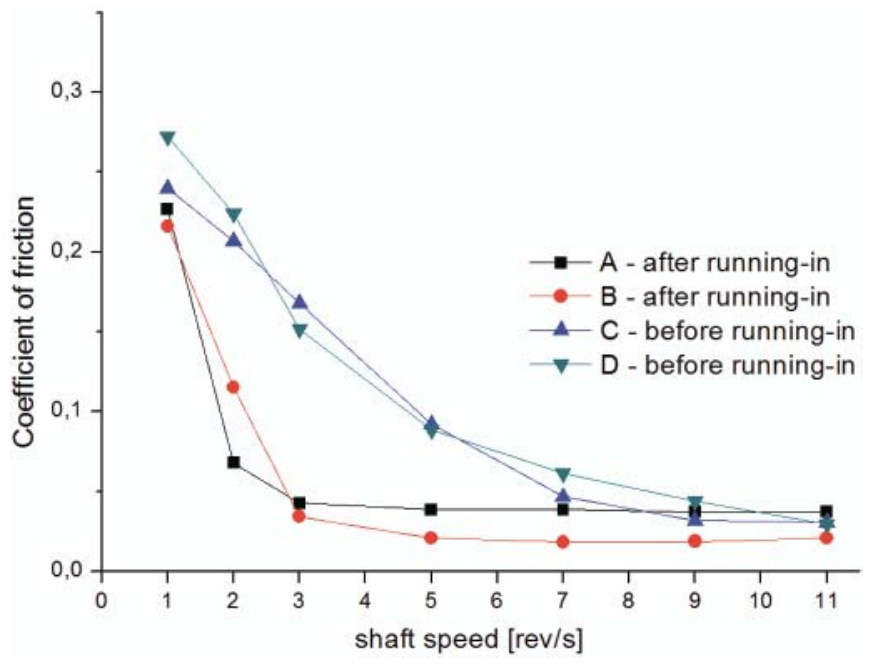

Fig. 1. Experimentally obtained operational characteristics of sliding bearing in function of shaft rotational speed for the bearings before, $(A, B)$; and after, $(B, C)$; running-in process

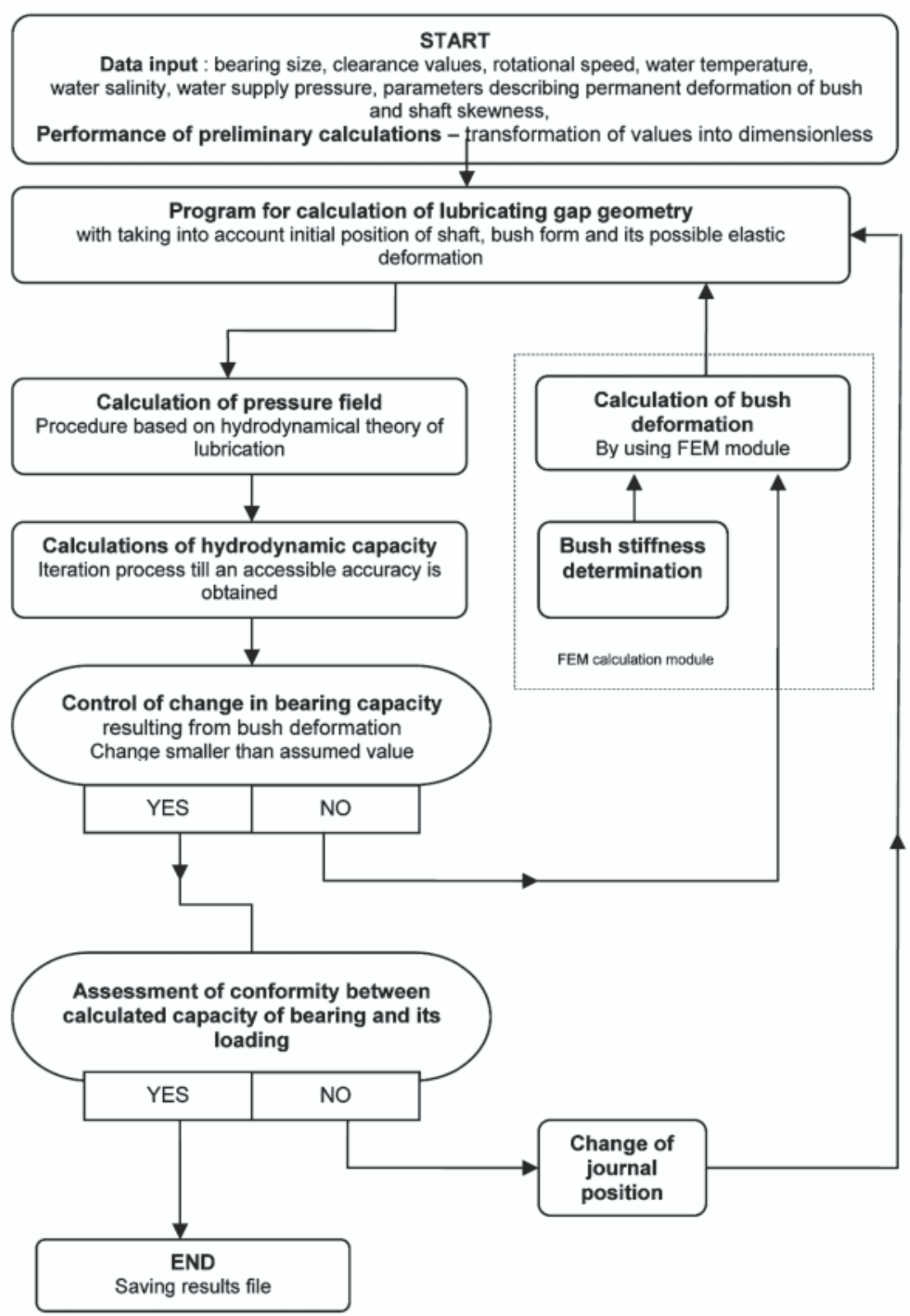

Fig. 2. Schematic diagram of the computation program 


\section{CALCULATIONS}

For calculations the elasto-hydrodynamic model (EHL) was used. The applied software was composed of two modules. The first of them was intended for the calculating of pressure distribution in lubricating film and based on hydrodynamical theory of lubrication and Reynolds equation. The other made it possible to calculate bush elastic deformation with the use of the finite element method (FEM).

The computational model was verified experimentally. The example calculated pressure distributions set against those from measurements are presented in Fig. 3 and 4. There are at least two reasons as to why certain differences between the results of the calculations and measurements occur. Probably both the measurements and calculations are loaded with an error. The measurement errors may result from an imperfect shape of the bush. The calculation results may be loaded with an error resulting from the assumed constant value of the material elasticity modulus amounting to only $800 \mathrm{MPa}$ in the case in question.

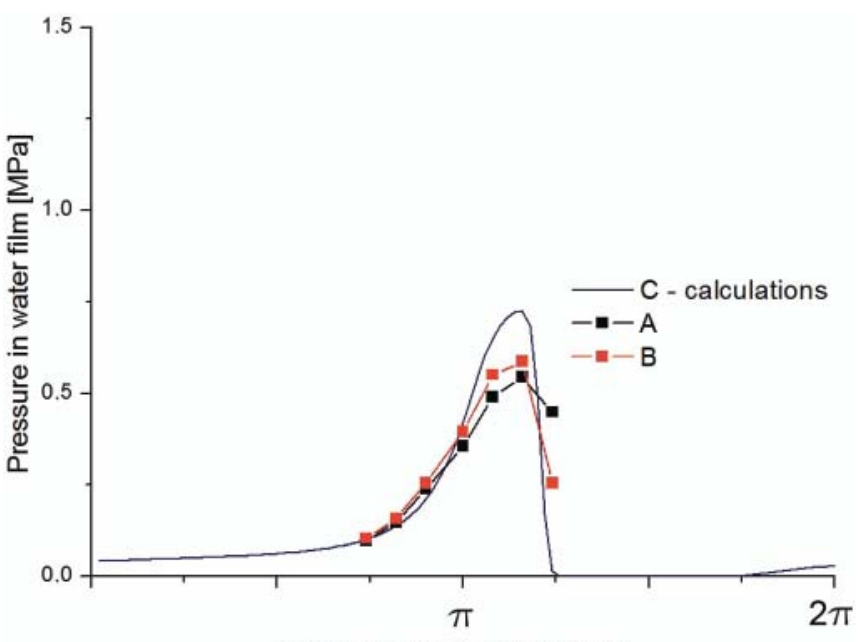

circumferential direction (rad)

Fig. 3. Pressure distribution in circumferential direction at bearing centerline; calculation results $(C)$ set against measurement results obtained on the test rig for the bearing with elastic bush $(A, B)$; for shaft diameter of $100[\mathrm{~mm}]$, rotational speed of 11 [rps], load of 0.1 [MPa]

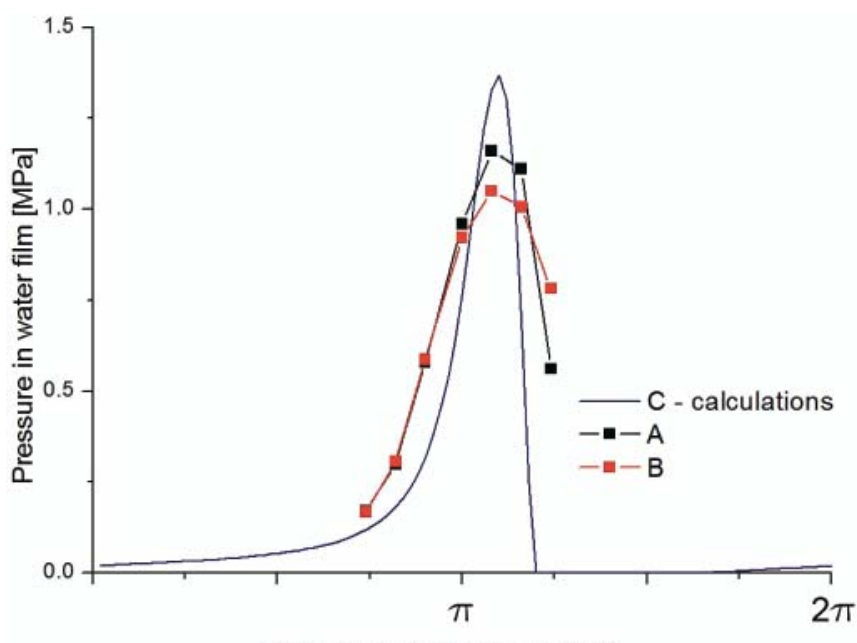

circumferential direction (rad)

Fig. 4. Pressure distribution in circumferential direction at bearing centerline; calculation results $(C)$ set against measurement results obtained on the test rig for the bearing with elastic bush $(A, B)$; for shaft diameter of $100[\mathrm{~mm}]$, rotational speed of 11 [rps], load of 0.2 [MPa]

All the below showed calculation results were obtained for the bearings similar to those experimentally tested. The shaft diameter was equal to $100 \mathrm{~mm}$, outer diameter of the bush $-124 \mathrm{~mm}$, length of the bush $-400 \mathrm{~mm}$ in compliance with the length/diameter ratio $\mathrm{L} / \mathrm{D}=4$, typical for ship shaft lines. The calculations were conducted for two values of shaft rotational speed: 3 and 11 rps.

\section{IMPACT OF VALUE OF BEARING CLEARANCE AND KIND OF BUSH MATERIAL ON BEARING OPERATIONAL CONDITIONS}

One of the crucial design parameters of sliding bearing is its diameter clearance. Authors of subject-matter publications usually make use of the so-called relative clearance whose value for water-lubricated polymer bearings generally ranges from 0.3 to $0.4 \%$ of shaft diameter and depends a.o. on shaft rotational speed. The usually suggested value of the clearance results from the general phenomenon of water absorbing by polymer, which leads to a reduction of internal diameter of bush, that is capable of full blocking the shaft in the extreme case. As results from catalogue data of various polymers the water absorption process may last from a few hours to even a few dozen weeks. This can be very dangerous as shaft seizing can theoretically happen just after ship's docking and even a dozen or so days after ship's departing from shipyard. The propeller shaft seizing and thus making the ship disabled may lead even to a catastrophe of fatal consequences. The water absorbing process of bush has been several times observed during the tests. Polymer is theoretically capable of absorbing a significant amount of water, and its volume increase may reach $6 \%$, but in practice even $0.1 \%$ value may be dangerous in view of reducing bush diameter even by a few tenth part of millimeter. Typical polymers applicable to shipbuilding increase their volume in the range from 0.1 to $0.5 \%$. In the past, when on the single bearing test stand a polyamide bush happened to be seized, it took almost three months to be able to dry it and then dismount the shaft.

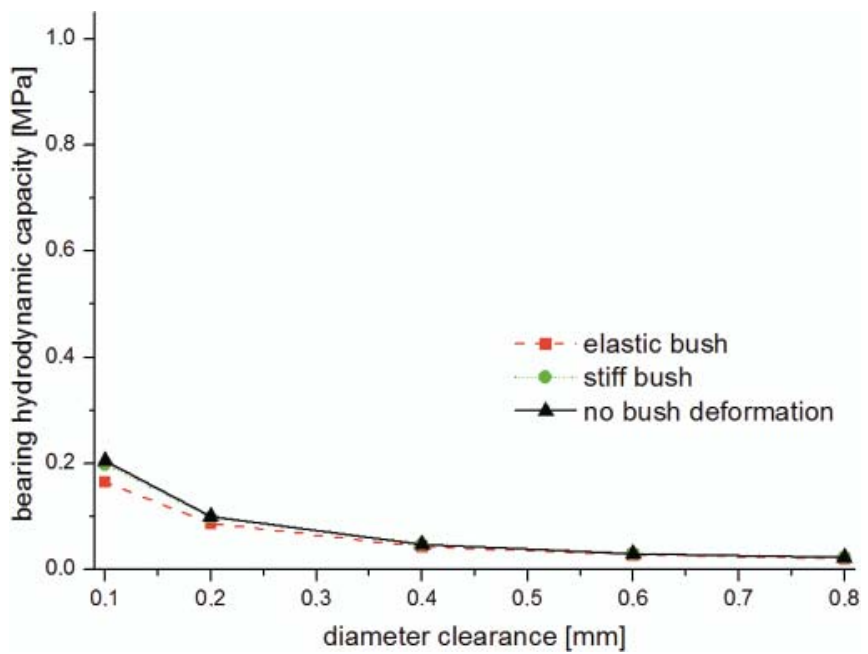

Fig. 5. The maximum theoretical hydrodynamical load-carrying capacity of the bearing fitted with the full bush of $100 \mathrm{~mm}$ shaft diameter, calculated for 3 rps shaft rotational speed

To determine impact of bearing clearance value on minimum theoretical hydrodynamical load-carrying capacity of bearing, calculations were performed under the assumption that the minimum acceptable value of lubricating film thickness amounts to $5 \mu \mathrm{m}$ (Fig. 5 and 6). The value may seem small as compared with that for classical oil-lubricated bearings but as experimental tests demonstrated polymer bearings are capable of stable operating at much thinner lubricating films. 
Measurements of drag to motion show bearing work in the fluid friction regime when to estimate value of lubricating film thickness on the basis of shaft axis trajectory and measured clearance circle is already not possible.

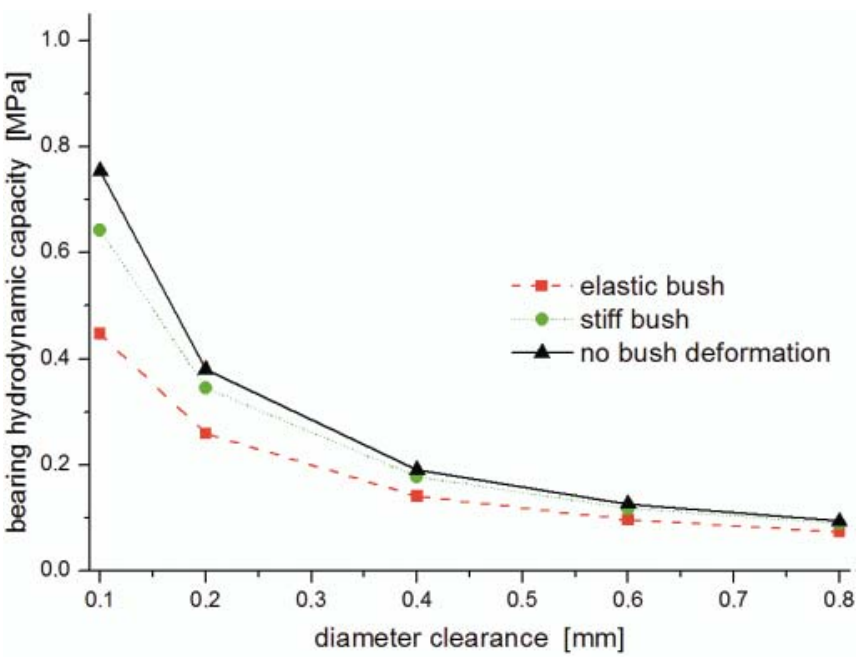

Fig. 6. The maximum theoretical hydrodynamical load-carrying capacity of the bearing fitted with the full bush of $100 \mathrm{~mm}$ shaft diameter, calculated for 11 rps shaft rotational speed.

The performed calculations show detrimental impact of increasing the bearing clearance, that greatly reduces potential, theoretical maximum hydrodynamical load-carrying capacity. Analyzing the diagrams one can conclude that for the shaft of the analyzed diameter the recommended clearance value which ranges from 0.3 to $0.4 \mathrm{~mm}$, is too large. Having at one's disposal a polymer of high form stability one is able to design a bearing of much better properties, for operating at relatively greater values of lubricating film thickness in the same conditions.

It is interesting that maximum load-carrying capacity of bearing decreases along with decreasing bush material elasticity (lower modulus of elasticity). However the opposite opinion can be often met. Accordingly, sometimes is stated that an elastic polymer material would operate better under fluid friction than highly stiff one, e.g. ceramics. To disqualify the thesis one should examine the hydrodynamical pressure distribution in a sliding bearing and its impact on elastic bush surface, presented in Fig. $7 \div 10$.

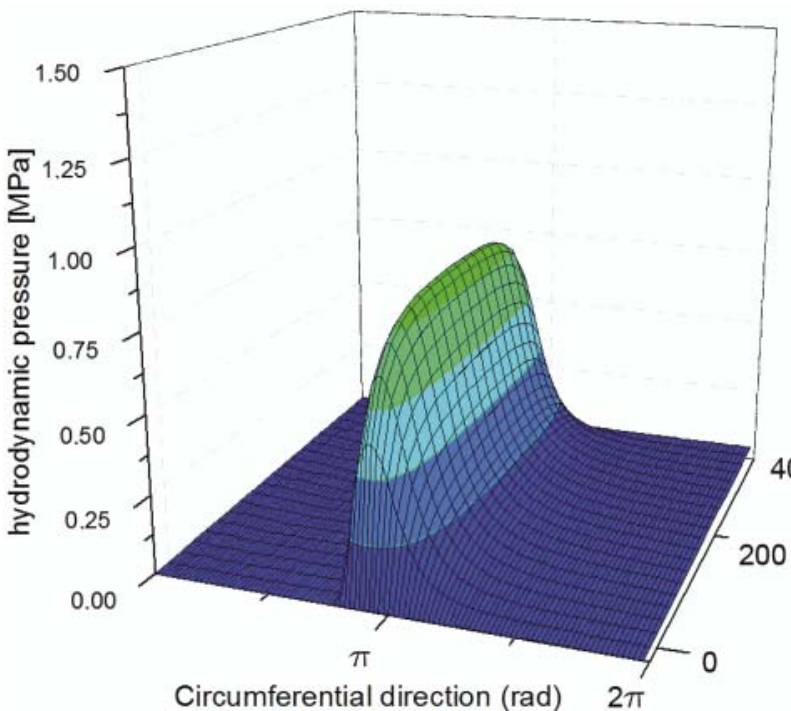

Fig. 7. The calculated pressure distribution in a highly loaded sliding bearing with elastic bush: for shaft diameter of 100 [mm], diameter clearance of 0.4 [mm], lubricating film thickness of 5 [ $\mathrm{\mu m}]$

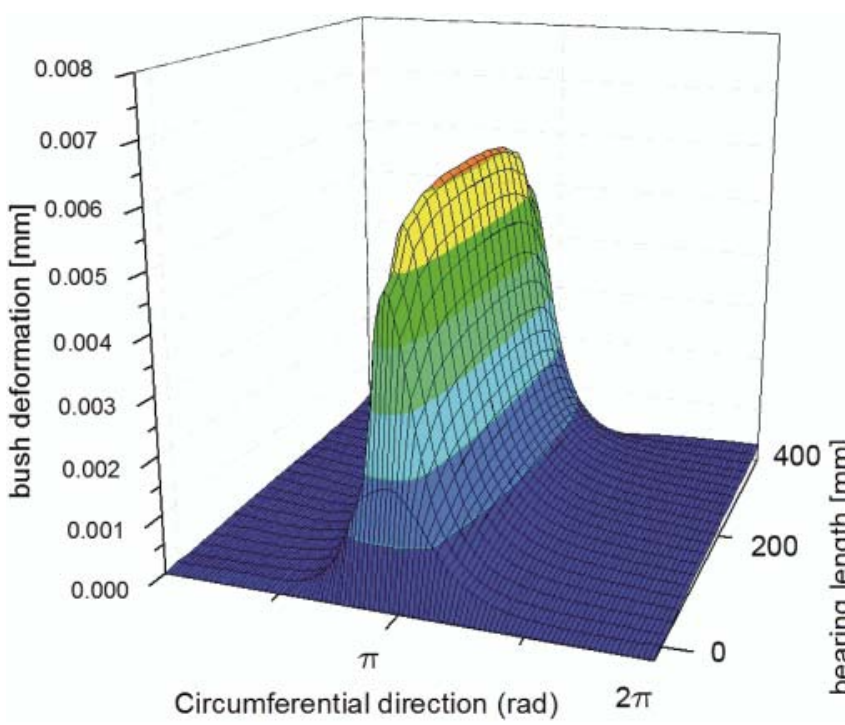

Fig. 8. The calculated bush surface deformation in a highly loaded sliding bearing with elastic bush; for shaft diameter of 100 [mm], diameter clearance of $0.4[\mathrm{~mm}]$, lubricating film thickness of $5[\mu \mathrm{m}]$

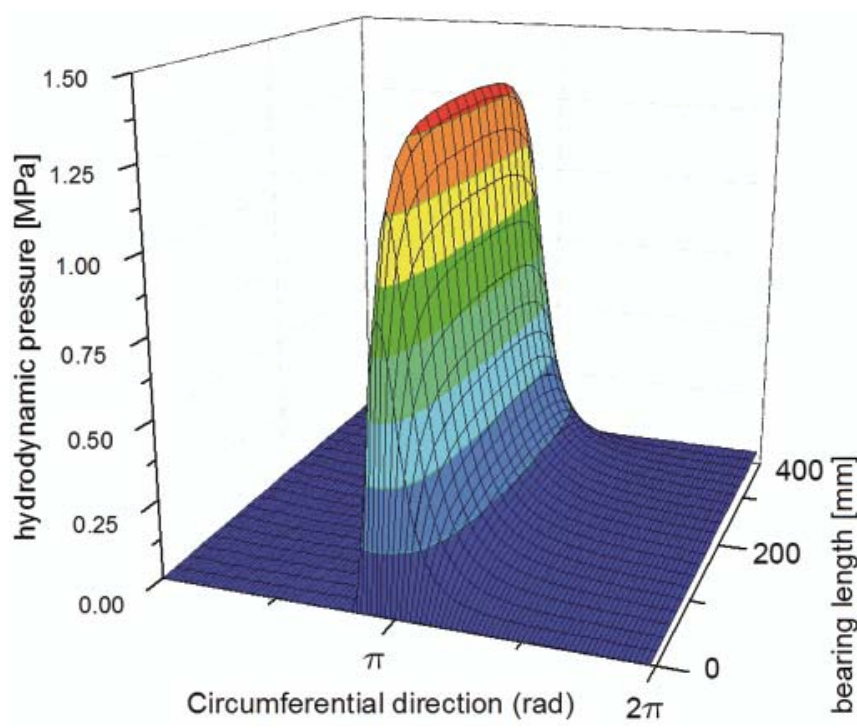

Fig. 9. The calculated pressure distribution in a highly loaded sliding bearing with stiff composite bush; for shaft diameter of 100 [mm], diameter clearance of 0.4 [mm], lubricating film thickness of $5[\mu \mathrm{m}]$

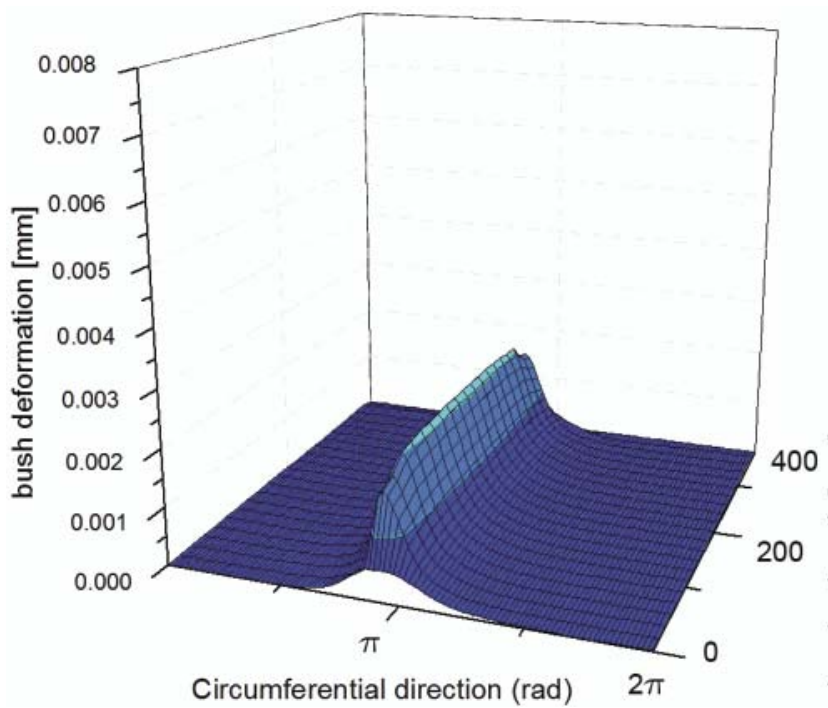

Fig. 10. The calculated bush surface deformation in a highly loaded sliding bearing with stiff composite bush; for shaft diameter of 100 [mm], diameter clearance of 0.4 [mm], lubricating film thickness of 5 [ $\mu \mathrm{m}]$ 
Calculating hydrodynamical sliding bearing one assumes lack of contact between its journal and bush. In order to calculate the maximum theoretical load-carrying capacity of the bearing one assumes a given minimum, but still acceptable, value of lubricating film thickness. Hence it turns out that assuming a more elastic bush material one makes that area of bush deformation is extended and reaches $7[\mu \mathrm{m}]$ in the case in question (Fig. 8), i.e. more than the assumed value of lubricating film thickness. That is why in reality the minimum gap height in the middle of the elastic polymer bush amounts to about $12[\mu \mathrm{m}]$. It led to lowering pressure in the film as compared with the more stiff composite material in which the deformation amounted to a little more than $2 \mu \mathrm{m}$ and the maximum pressure in the film reached about $1.5 \mathrm{MPa}$ (Fig. 9 and 10). Basing on the calculations one can state that gradually increasing loading of the bearings fitted with elastic bush would result in gradual transition from operation under full hydrodynamical lubrication to that under partial fluid friction. A contact zone will appear in the area close to both edges of bush where hydrodynamical pressure is negligible and in consequence deformation is small. Along with gradual load increasing the fluid friction zone will be smaller and smaller and finally disappear when the bearing starts operating entirely in the mixed friction regime. The thesis is confirmed by experimental tests which show the bearings to operate under fluid friction. Analysis of the tested bushes revealed also a significant wear in the contact zone close to outer edges of the investigated bearings.

The next important factor of a great impact on operation of hydrodynamical sliding bearing is kind and temperature of water flowing through the bearing. It is particularly important for open lubricating systems where outboard water is used as a lubricating medium. The case can be assumed when a ship passes from warm fresh waters of Mississippi river to cold waters of North Canada. Then water temperature drop may reach about $20^{\circ} \mathrm{C}$ and water viscosity may be additionally increased by salt contained in sea water. Hence the dynamical viscosity increase from about 890.2 to $1604.6[\mathrm{~Pa} \cdot \mathrm{s}]$ may be expected [2]. To assess viscosity change influence on maximum theoretical load-carrying capacity of bearing appropriate calculations were conducted whose results have been presented below (Fig. 11).

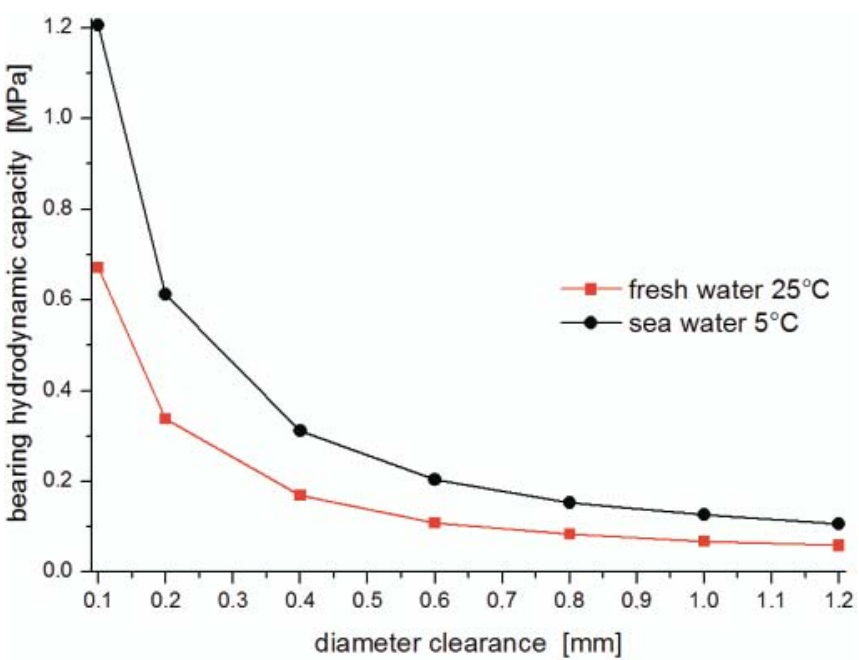

Fig. 11. The calculated maximum theoretical load-carrying capacity of the bearing with full bush; for 100mm shaft diameter, shaft rotational speed of 11 [rps] and two different values of water viscosity:890,2 and 1604,6 [Pa:s]

The results showed that especially in the range of small values of diameter clearance theoretical capacity values could differ almost twofold. During sailing on the analyzed route, if only the bearing operates under fluid friction, film thickness values will be increasing along with temperature dropping and growing salinity of the waters. Also, the possible cooling of water used for lubrication of bearings operating in closed system, may be considered. In this case operational conditions of bearings could be much improved at the expense of a greater energy demand. That is why the solution proposed by some producers to use water from engine's cooling system for lubrication of bearings seems very unfavourable.

\section{IMPACT OF LUBRICATING GROOVES ON BEARING PROPERTIES}

Water-lubricated sliding bearings fitted with polymer bush in which longitudinal, passing-through lubricating grooves are made, are the typical solution used not only in shipbuilding but also in water power plants and pumps. As already mentioned, it results first of all from the necessity of effective absorbing heat from friction zone as polymer bush does not transfer heat to casing and is additionally sensitive to elevated temperature [3]. Arrangement of the grooves round bush circumference makes it possible to increase rate of water flow through bearing and allows this way to improve cooling conditions. In practice it turns out that the grooves fulfil one very important function more as wear products and various contaminations sediment in them. It is of particular importance for the simplest propeller shaft bearing systems, the open ones where during ship sailing e.g. in silt-up, shallow inland waterways a large quantity of various contaminations may fall into bearing's interior. The cases are known when even small crustaceans set down inside lubricating grooves.

Studying the subject-matter literature, in particular guidelines for application of various sliding materials, published by worldwide producers, one can conclude that they are very similar to each other. Producers selling prefabricates usually in the form of pipes, make their guidelines first of all directed to designers, in which are included many necessary data such as: recommended values of bearing clearance and thermal shrinkage as well as number, size and arrangement of lubricating grooves. From the publications it can be concluded that two concepts dominate. According to the first of them it is proposed to arrange grooves round entire bush circumference (Fig. 12. variant A). The other concept follows the hydrodynamical lubrication theory. Therefore its authors came to the conclusion that it is not recommended to place grooves in the lower part of bearing as it could limit its potential capabilities (Fig. 12. variant C).

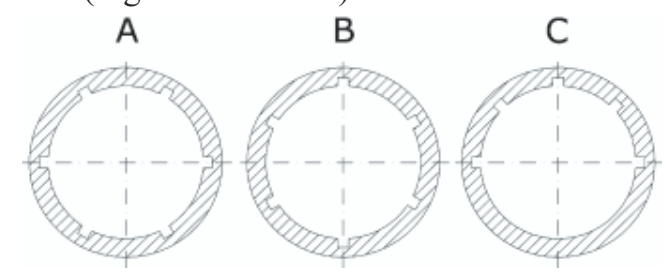

Fig. 12. Various practically used arrangements of lubricating grooves within bush; $\boldsymbol{A}$ - recommended arrangement of many grooves round entire bush circumference, $\boldsymbol{B}$ - erroneous position of bush, $\boldsymbol{C}$ - bush with grooves located only round the upper half of bush.

The performed calculations of water-lubricated hydrodynamical sliding bearing fitted with longitudinal open lubricating grooves have demonstrated that their arrangement greatly impacts properties of the bearing. Moreover the calculation results have proved that the proper arrangement of grooves is not of a detrimental effect to the bearing properties (Fig. 13). This results from the influence of grooves on the forming of lubricating film in the bearing. As far as the bearing 
fitted with full bush is concerned a gradual hydrodynamic pressure increase takes place within the film (Fig. 15).

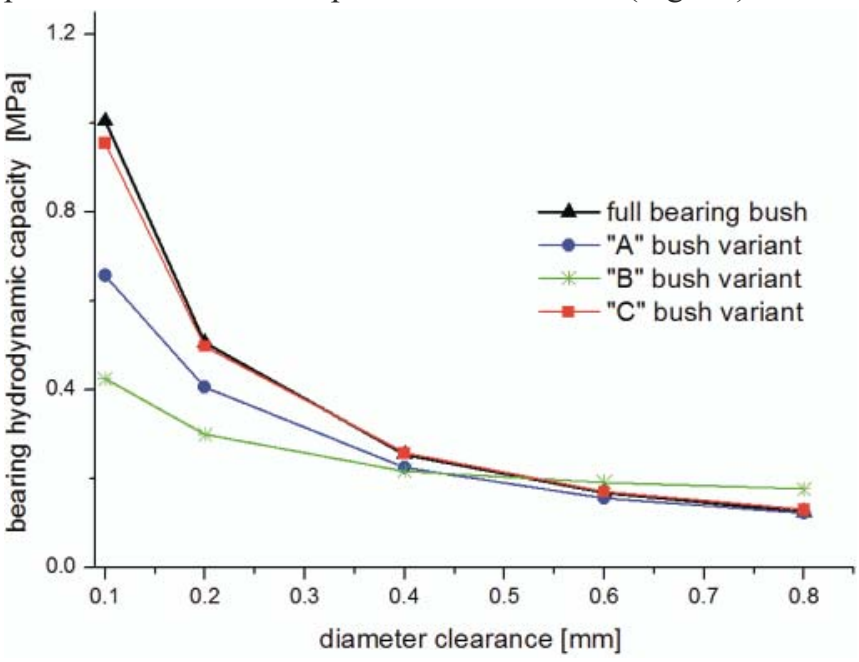

Fig. 13. The calculated maximum load-carrying capacity of bush in function of bearing clearance; for shaft diameter of $100 \mathrm{~mm}$, shaft rotational speed of 11 [ rps], fresh water of $7^{\circ} \mathrm{C}$ temperature, $\eta=1.43[\mathrm{kPa} \cdot \mathrm{s}]$

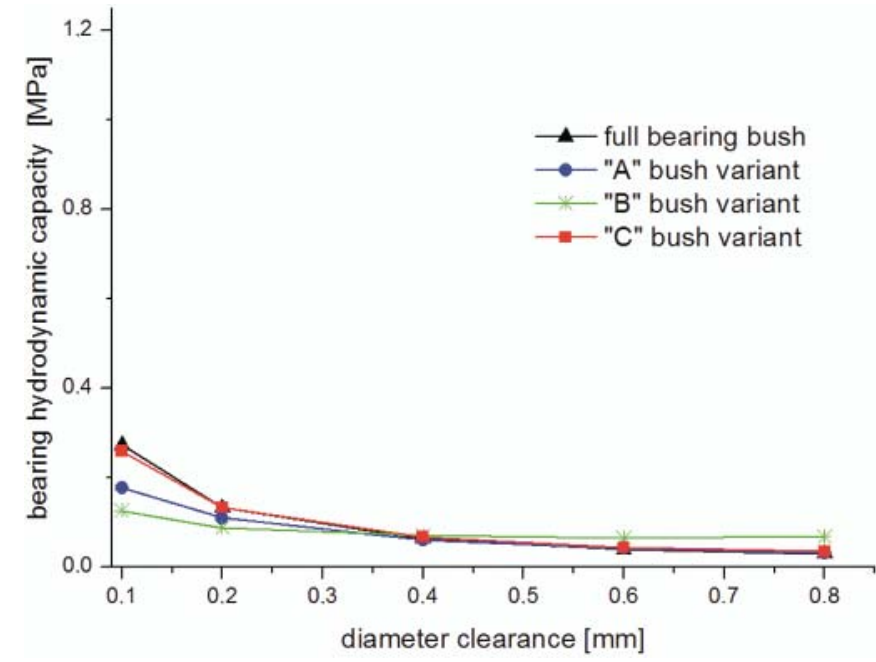

Fig. 14. The calculated maximum load-carrying capacity of bush in function of bearing clearance; for shaft diameter of $100 \mathrm{~mm}$, shaft rotational speed of 3 [ rps], fresh water of $7^{\circ} \mathrm{C}$ temperature, $\eta=1.43[\mathrm{kPa} \cdot \mathrm{s}]$
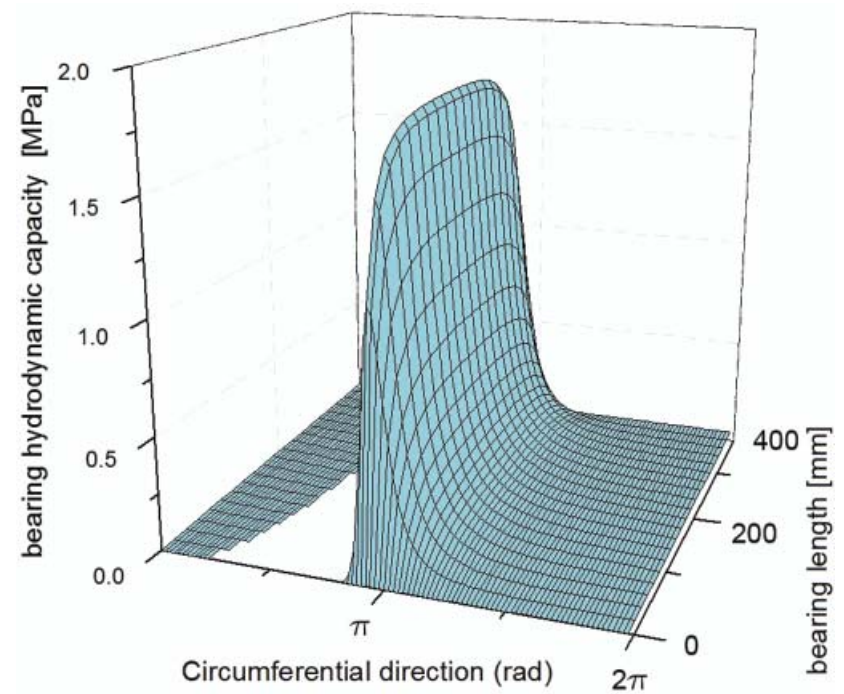

Fig. 15. The calculated pressure distribution in lubricating film of sliding bearing of full bush; for shaft diameter of 100 [mm], rotational speed of 11 [ rps], load-carrying capacity of 15.2 [kN], fresh water at $7\left[{ }^{\circ} \mathrm{C}\right], \eta=1,43[\mathrm{kPa} \cdot \mathrm{s}]$

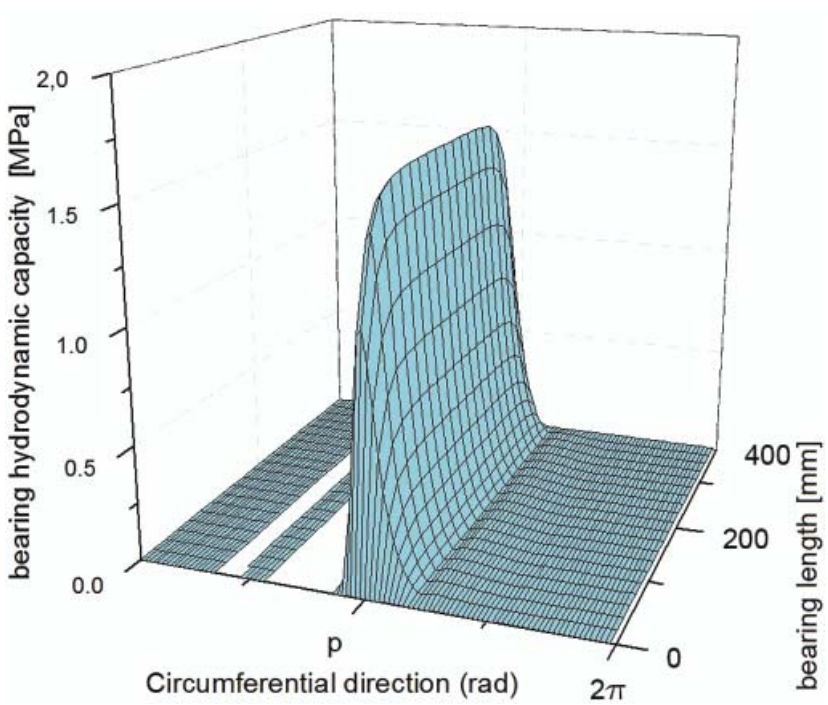

Fig. 16. The calculated pressure distribution in lubricating film of sliding bearing having six grooves of A variant; for shaft diameter of 100 [mm], rotational speed of 11 [ rps], load-carrying capacity of 12.2 [kN],

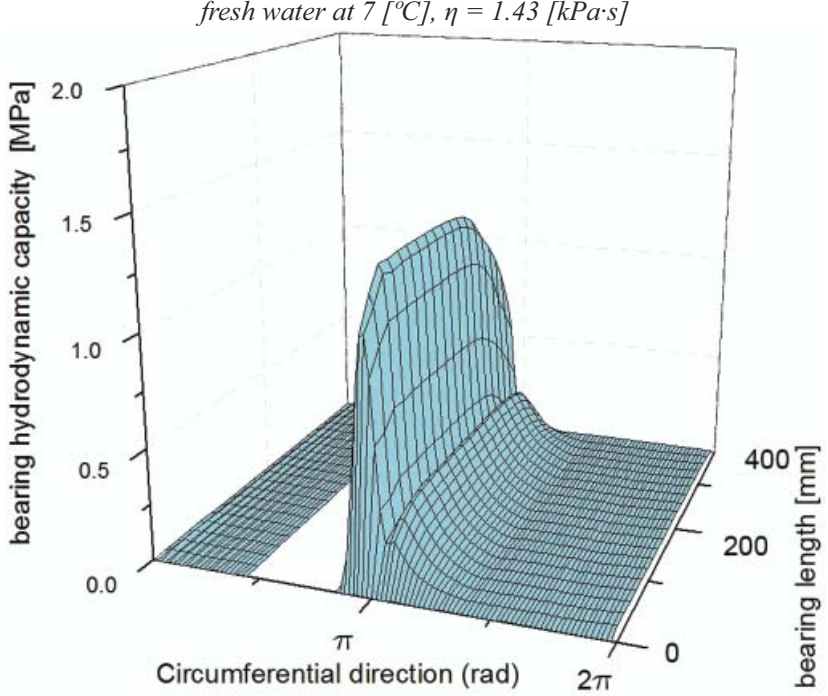

Fig. 17. The calculated pressure distribution in lubricating film of sliding bearing having six grooves of B variant; for shaft diameter of $100[\mathrm{~mm}]$, rotational speed of 11 [ rps], load-carrying capacity of 9 [kN],

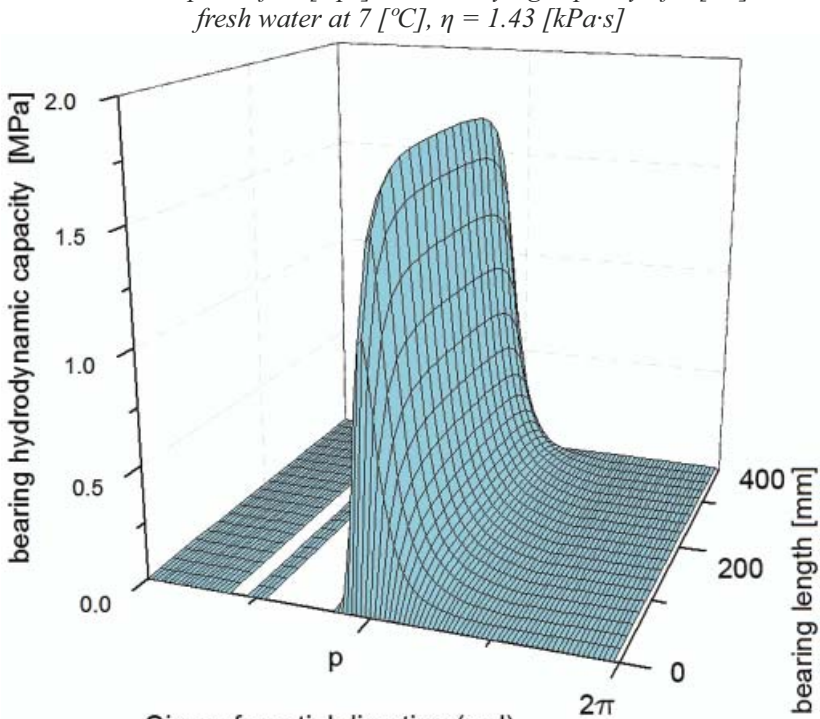

Circumferential direction (rad)

Fig. 18. The calculated pressure distribution in lubricating film of sliding bearing having six grooves of C variant; for shaft diameter of 100 [mm], rotational speed of 11 [ rps], load-carrying capacity of 15 [kN], fresh water at $7\left[{ }^{\circ} \mathrm{C}\right], \eta=1.43[\mathrm{kPa} \cdot \mathrm{s}]$ 
The phenomenon is disturbed by lubricating grooves in which the so called solution of lubricating film continuity occurs. Therefore behind the groove the pressure starts to grow again but its increase is usually more violent (Fig. 16). In the worst situation when the grooves are especially unfavourable located a definite reduction of bearing capacity may happen and the pressure increase zone within lubricating film may be split into two parts (Fig. 17). Therefore the optimum solution for ship propeller shafts in which the load generated by rotating mass of the shaft-propeller unit exerts mainly the lower part of bush, consists in leaving the lower part of bush free of lubricating grooves (Fig. 18).

\section{RECAPITULATION}

- The ship propeller - shaft water-lubricated bearings fitted with polymer bushes are very sensitive elements. However, if properly designed and assembled, they are able to operate reliably for a long time. They are capable of working under fluid friction in spite of that they are lubricated with low-viscosity liquid. However they show much lower load-carrying capacity as compared with oil-lubricated bearings. In water-lubricated bearings value of lubricating film thickness is usually small and reaches no more than a few micrometers. Hence in typical cases such bearings do not have too large margin of hydrodynamical load-carrying capacity, moreover possible errors in their shape and shaft alignment, especially skewness, make that the bearings would operate in the mixed friction regime.

- As the performed investigations demonstrated the effective method for improving bearing capacity is to limit its clearance value. However is should be remembered that polymers generally absorb water and in consequence enlarge their volume, that may cause power shaft seizing.

- An effective method to increase potential load - carrying capacity of bearing is to apply a stiff composite material to its bush. However it should be taken into account that the bearing will become then greatly sensitive to errors in bush shape and shaft alignment.
- Mechanism of delamination failures in composite bushes is not fully recognized. During many experimental tests have been performed so far it was not possible to generate such failure. To cause it are deemed the local superheating of bearing or propulsion shaft vibrations. Hence in designing a propulsion system of the kind appropriate heat exchange conditions should be ensured. It is especially important for the bearings with polymer bush as heat exchange through casing does not occur in them. A prevailing amount of heat generated in friction zone is to be absorbed by lubricating medium. Therefore in most cases grooves are made in bushes, owing to that it is possible to increase rate of water flow through the bearing. In the typical case, as results from the performed tests, the grooves should be placed only in the upper part of bush in consequence they do not have practically any detrimental effect to hydrodynamical properties of bearing. It should be added that the grooves play also another important function as within them bearing wear products and contaminations can be sedimented.

\section{BIBLIOGRAPHY}

1. Litwin W.: Marine water lubricated main shaft bearings, problems, theoretical and experimental research; Polish Maritime Research no 4/2009, Vol. 16

2. Litwin W.: Water - An Extraordinary, Ordinary Lubrication Liquid. Influence Of Water Salinity, Pressure And Temperature On Water Lubricated Bearings Properties; ASME/STLE International Joint Tribology Conference, October 17-20, 2010, San Francisco, California, USA

3. Rymuza Z.: Tribology of sliding polymers (in Polish). WNT (Scientific Technical Publishing House)Warsaw 1986.

\section{CONTACT WITH THE AUTHOR}

Wojciech Litwin, Ph. D.

Faculty of Ocean Engineering and Ship Technology

Gdansk University of Technology Narutowicza 11/12 80-233 Gdansk, POLAND e-mail: wlitwin@pg.gda.pl 